\title{
INICIAÇÃO CIENTÍFICA: UMA ANÁLISE DE SUA CONTRIBUIÇÃO NA FORMAÇÃO ACADÊMICA
}

\author{
Maria Janice Pereira Lopes* \\ Dárcio Luiz de Sousa Júnior ${ }^{* *}$
}

RESUMO: A iniciação científica é um programa desenvolvido nas Instituições de Ensino Superior que permite introduzir estudantes da graduação na pesquisa científica, servindo de apoio técnico e metodológico para a sua formação. A finalidade deste programa é promover o desenvolvimento do estudante-pesquisador, o qual praticará os primeiros momentos da pesquisa acadêmica, dando-lhe maturidade intelectual e desenvolvendo sua compreensão em relação à ciência concedendo-lhe possibilidades futuras, tanto acadêmicas quanto profissionais. O objetivo central deste trabalho foi o de compreender a contribuição da iniciação científica na formação acadêmica, por meio de uma pesquisa bibliográfica em teses, dissertações, artigos e trabalhos apresentados em congresso e/ou simpósio que abordaram o tema. Concluindo que a iniciação cientifica traz inúmeros benefícios aos jovens universitários, ao se constituir em um excelente instrumento educativo possibilitando ao estudante a participação do processo de produção do conhecimento científico de sua área de formação, melhores desempenhos nas seleções para a pós-graduação, espírito de equipe, mais facilidade de falar em público e de se adaptar às atividades didáticas, transforma-o de agente passivo para ativo, permite-lhe uma evolução que implicará em resultados positivos para a universidade e para a comunidade em que este estudante esteja inserido.

PALAVRAS-CHAVE: Ensino; Graduação; Iniciação Científica.

\section{SCIENTIFIC INITIATION: AN ANALYSIS OF ITS CONTRIBUTION IN ACADEMIC FORMATION}

ABSTRACT: Scientific initiation is a program developed by Institutions of Higher Education to introduce undergraduate students to scientific research, coupled to technique and methodological support for their formation. The program aims at developing the student-researchers who will start practicing academic research, provi-

Farmacêutica, Especialista em Docência e Gestão do Ensino Superior, Departamento de Biofisiologia e Farmacologia da Faculdade de Medicina Estácio de Juazeiro do Norte, Ceará, Brasil.

E-mail: jannicelopes@hotmail.com

* Biomédico, Especialista em Microbiologia Clínica, Departamento de Biofisiologia e Farmacologia da Faculdade de Medicina Estácio de Juazeiro do Norte, Ceará, Brasil. 
ding them with intellectual maturity and developing their understanding on science for a better academic and professional future. Current paper deals with the contribution of scientific initiation in academic formation through a bibliographic research in theses, dissertations, articles and papers in congresses and symposia on the theme. Results show that scientific initiation brings numberless benefits to young students. It is an excellent educational tool which makes possible the students' participation in the process of scientific production in the area of formation, better performances in selections for postgraduate candidates, team spirit, facility to speak in public and adaptation to didactic activities. The activities transforms the agents into active ones, providing them which an evolution that implies in positive results for the university and the community in which they are inserted.

KEY WORDS: Teaching; Undergraduate; Scientific Initiation.

\section{INTRODUÇÃO}

A Iniciação Científica (IC) é um programa desenvolvido nas Instituições de Ensino Superior (IES) que permite inserir estudantes da graduação na pesquisa científica, servindo de apoio técnico e metodológico à sua formação. Ao ingressar na IC o aluno vivencia experiências vinculadas a um projeto de pesquisa, elaborado e desenvolvido sob a orientação de um docente (SIMÃO et al., 1996). Esta modalidade de pesquisa acadêmica serve como instrumento de apoio teórico e metodológico que potencializa as chances de um estudante de graduação engajar na pesquisa científica, auxiliando-o no desenvolvimento das visões do aluno, permitindo-o um contato com o conhecimento superior ao visto em sala de aula. A IC deve ser vislumbrada como um componente essencial de formação (OLIVEIRA, 2017).

A finalidade deste programa é promover o desenvolvimento do estudante -pesquisador, o qual praticará os primeiros momentos da pesquisa acadêmica, como a escrita científica, apresentação de resultados em eventos, elaboração de relatórios e demais atividades envolvendo o ofício de pesquisador. É uma oportunidade para os estudantes desenvolverem suas habilidades acadêmicas e interpessoais, bem como encontrarem direcionamento e apoio profissional (PINTO; FERNANDES; SILVA, 2016).

Atualmente, com a globalização, o mercado cada vez mais competitivo e 
o surgimento de novas tecnologias há uma maior exigência por profissionais que possuam conhecimento teórico e prático baseada na reprodução e produção de conhecimentos (CAMPOS; SANTOS; SANTOS, 2009). A sociedade exige qualificações cada vez mais elevadas, ampliando-se as necessidades educacionais da população. Diante desse cenário, cresce a importância dos cursos de graduação e o desenvolvimento das capacidades para adaptar-se às mudanças, procurando continuamente novos conhecimentos e soluções para os problemas do dia a dia, especialmente os relacionados ao mundo moderno e tecnológico, trazendo assim progresso para a ciência e para a sociedade (SANTOS, 2011), e a universidade, juntamente com os professores, tem um papel de destaque nesse processo.

O objetivo central deste trabalho foi o de compreender a contribuição da iniciação científica na formação acadêmica.

A principal justificativa para a realização deste estudo foi reforçar o valor da iniciação científica para a formação acadêmica, por meio de informações que comprovem o quanto o estudante-pesquisador se destaca pessoal e profissionalmente quando desenvolve pesquisa científica.

A metodologia utilizada foi uma pesquisa bibliográfica em teses, dissertações, artigos e trabalhos apresentados em congresso e/ou simpósio que abordaram o tema.

\subsection{INICIAÇÃO CIENTÍ́FICA}

A iniciação científica é um processo no qual é fornecido um conjunto de conhecimentos indispensáveis para o estudante iniciar os ritos, técnicas e tradições da ciência, ou seja, é uma porta de entrada para conhecerem o campo do trabalho acadêmico, da mesma forma em que simboliza uma oportunidade para as instituições de ensino e pesquisa atraírem novos talentos (MASSI; QUEIROZ, 2010).

Segundo Oliveira (2017), a IC é um instrumento que permite a inserção de estudantes de graduação potencialmente mais promissores na pesquisa, possibilitando-os desde cedo o contato direto com a atividade científica, desenvolvendo suas aptidões, bem como influenciando-os no desenvolvimento crítico, ético e cultural, preparando-os com excelência para o mercado de trabalho.

Ao participar do programa de IC os alunos têm a chance de realizarem e par- 
ticiparem do processo de produção do conhecimento, beneficiando-se tanto com o desenvolvimento de uma atividade de pesquisa, quanto com a própria entrada em programas oficiais que lhes possibilitem contato com um orientador qualificado, aquisição de conhecimento científico e informação de uma área específica, além de lhes proporcionar remuneração (BRIDI, 2004).

Este processo ocorre por meio das múltiplas atividades desenvolvidas durante os anos da graduação, tais como; aulas teóricas e práticas, palestras, participação em projetos experimentais e de pesquisa, monitorias, elaboração de trabalhos monográficos, estudos individuais e em grupos, participação em congressos e eventos científicos etc. Estas atividades devem estar alicerçadas em um projeto pedagógico e por meio delas vai sendo construído um determinado perfil profissional (OLIVEIRA, 2001).

De acordo com o Conselho Nacional de Desenvolvimento Científico e Tecnológico - CNPq (2017), a iniciação científica tem a finalidade de elevar o patamar de informações disponíveis e a popularização da ciência e da tecnologia como conhecimentos essenciais a todos.

\subsection{INICIAÇÃO CIENTÍ́IICA NO BRASIL}

Para criar o Programa de Iniciação Científica (PIC), as Universidades Brasileiras foram buscar inspiração nos países que já tinham uma atividade científica institucionalizada: Estados Unidos e França. Nos EUA, o programa Research and Development envolve os alunos de ciência e engenharia na produção de uma tese relacionada às atividades em curso no Departamento no último ano de graduação. $\mathrm{Na}$ França, a atividade, menos formal, consiste em estágio em um laboratório universitário ou industrial, a partir do qual o estudante apresenta um relatório final (BAZIN, 1983).

No Brasil, os primeiros programas de IC datam da primeira metade do século XX, porém os programas de fomento e financiamento oficializados surgiram apenas em 1951, com a criação do CNPq (MASSI; QUEIROZ, 2010), que abriu oportunidade de apoio financeiro a pesquisadores por meio de bolsa, obtidas pelo orientador, as quais eram concedidas aos alunos de graduação (SILVA, 2012).

Até 1988 as bolsas de IC só podiam ser distribuídas mediante solicitação di- 
reta do pesquisador, essas eram denominadas 'bolsas por demanda espontânea' ou 'balcão'. Os pedidos eram julgados por Comitês Assessores e concedidos por cotas aos pesquisadores, que escolhiam os bolsistas (MASSI; QUEIROZ, 2015).

Ao observar a baixa abrangência dessa modalidade de fomento, o CNPq criou, em 1988, o Programa Institucional de Bolsas de Iniciação Científica (PIBIC), por meio do qual as bolsas de IC também eram concedidas diretamente às Instituições de Ensino Superior e aos Institutos de Pesquisa, que passaram a gerenciar as concessões dessas bolsas. Atualmente o CNPq mantém esses dois programas de bolsas (MASSI; QUEIROZ, 2014).

Em 1988 o processo deu uma grande guinada com a criação do PIBIC pelo CNPq, que passa a conceder bolsas para alunos de graduação desenvolvendo atividades de pesquisa. O sucesso do programa foi imediato e replicado pelas agências estaduais de fomento, como a Fundação de Amparo à Pesquisa do Estado de São Paulo (FAPESP), Fundação de Amparo à Pesquisa do Estado do Rio de Janeiro (FAPERJ), Fundação de Amparo à Pesquisa do Estado de Minas Gerais (FAPEMIG), entre outras (SILVA, 2012).

Para Sanches (2016), apesar de seu valor, existem diversos desafios relacionados à IC no Brasil atualmente, dentre eles a precariedade no financiamento, pois um país não faz ciência apenas investindo financeiramente em cientistas e laboratórios, esses investimentos são imprescindíveis, mas não são suficientes. É preciso ter em mente que o progresso está naquilo de positivo que a ciência pode oferecer a um país e à sua população.

Muitas instituições de ensino superior têm tido cada vez mais dificuldade em obter recursos para financiar suas pesquisas, devido aos crescentes cortes no número de bolsas concedidas, seja pela necessidade de financiar a compra de equipamentos, livros e serviços de manutenção para laboratórios. Fazer pesquisa não é barato e precisa de muito apoio institucional (ÂNIMA EDUCAÇÃO, 2017).

Essa redução no número de bolsas ocorre em todos os âmbitos do sistema superior de ensino atualmente, da graduação ao doutorado. Isso é um problema, já que a concessão de bolsas permite mais dedicação de pesquisadores ao objeto de estudo, bem como o engajamento em outras atividades acadêmicas. Quando não há bolsa, muitos alunos acabam preferindo realizar estágios remunerados fora da faculdade. E, além da falta de recursos, há uma burocracia sem fim que atravanca 
toda e qualquer iniciativa.

Para que o Brasil se torne uma potência científica, é necessária uma mudança profunda em toda sua estrutura, desde a desburocratização da política de exportação e importação de material científico até investimentos vindos da iniciativa privada. Em virtude das dificuldades em se realizar pesquisa científica no Brasil e em países em desenvolvimento, se presencia uma emigração de pesquisadores para países com tradição nesse segmento, as empresas brasileiras ainda não exploram o potencial da interação com institutos de pesquisa e universidades (SANCHES, 2016).

\section{O PAPEL DO PROFESSOR NO INCENTIVO À PESQUISA CIENTÍFICA}

Para ser professor é imprescindível que tenha uma formação específica para desenvolver determinadas competências e subsídios teórico-práticos para desempenhar a sua função. Não é suficiente que o professor tenha um grande domínio de seu conteúdo específico, é necessário que esse conteúdo esteja relacionado com o momento vivido pela sociedade (NETO; MACIEL, 2009).

O professor tem deixado de ser um mero transmissor de conhecimentos para ser mais um orientador, um estimulador de todos os processos que levam os alunos a construírem seus conceitos, valores, atitudes e habilidades que lhes permitam crescer como pessoas, como cidadãos e futuros trabalhadores, desempenhando uma influência verdadeiramente construtiva (SANTOS, 2017).

Os professores possuem uma interferência direta na maneira com que a pesquisa é/ou deveria ser incentivada aos alunos, pois são eles quem ministra as disciplinas, selecionam as estratégias, as formas de avaliação e detêm o poder de decidir se estimula ou não os hábitos investigativos (PELUSO, 2013). Afinal, ensinar é uma arte que necessita ser aperfeiçoada a cada dia, e a pesquisa faz parte disso (SANTOS; FERREIRA, 2014).

Segundo Santos (2017), o ensino não deve apenas formar trabalhadores para as exigências do mercado de trabalho, mas cidadãos críticos capazes de transformar um mercado de exploração em um mercado que valorize uma mercadoria cada vez mais importante: o conhecimento.

Dentro das competências: científica, técnica, humana e política desenvol- 
vidas pelo professor, é essencial propiciar aos alunos condições para o desenvolvimento da capacidade de pensar crítica e logicamente, fornecendo-lhes meios para a resolução dos problemas inerentes aos conteúdos trabalhados interligados ao seu cotidiano, fazendo com que ele compreenda que o estudo é mais do que mera memorização de conceitos e termos científicos transmitidos pelo professor ou encontrados em livros (SANTOS, 2017).

Neste sentido o professor na atualidade se define como orientador de todo o processo de aprendizagem do aluno acadêmico, e por muitas vezes ele é a fonte de inspiração, e deve assegurar a produtividade do ensino, ou seja, o professor precisa ser um aliado comprometido na constituição do indivíduo (SANTOS; FERREIRA, 2014).

\section{A INICIAÇÃO CIENTÍ́fICA NAS INSTITUIÇÕES DE ENSINO SUPERIOR}

As IES são consideradas um campo privilegiado para conhecer a cultura universal e as várias ciências, para criar e difundir o conhecimento. A função das IES, como instituição social, consiste em formar o estudante universitário de maneira sistemática em profissionais, técnicos, intelectuais e cientistas aptos a desenvolverem atividades profissionais qualificadas. É, ainda, espaço por excelência no qual acontecem trocas de informações, experiências e saberes, estabelecendo interação entre cientistas, técnicos, docentes e discentes (PIMENTA, 2005).

Segundo a DELIBERAÇÃO CONSU-A-24/03 do Fundo de Apoio ao Ensino, Pesquisa e Extensão é dever da Universidade "incentivar a pesquisa por todos os meios ao seu alcance, incluindo a concessão de auxílios para a execução de projetos específicos" (OLIVEIRA, 2008).

Cita ainda que: "Uma das prioridades da Universidade é a formação de recursos humanos capacitados para contribuir de modo efetivo no processo de desenvolvimento do País e, para tanto, é imprescindível uma atenção especial ao ensino de graduação e de pós-graduação”.

Por meio da IC a Universidade pode atingir todos estes objetivos acima citados, bem como ser divulgada e seu domínio de atividades transpor os limites físicos 
da instituição e se perpetuar através da transitividade do conhecimento oferecido.

E conforme a Lei de Diretrizes e Bases da Educação Nacional (Lei ${ }^{0}$ 9.394/1996) (BRASIL, 1996, p. 14), a universidade deve "incentivar o trabalho de pesquisa e investigação científica, visando o desenvolvimento da ciência e da tecnologia e da criação e difusão da cultura, e, desse modo, desenvolver o entendimento do homem e do meio em que vive". Sendo assim é um dever da universidade fomentar a pesquisa de forma a estimular os discentes a desenvolver o interesse pela iniciação científica e dar condições estruturais para a prática da produção científica. Assim a pesquisa deve ser vista como parte do programa de ensino da universidade (SANTOS, 2011).

As Instituições de Ensino têm o papel de contribuir com a sociedade por meio da produção de conhecimento pela pesquisa científica e pela formação de profissionais e cidadãos, promovendo a autonomia de pensamento e da reflexão crítica, não se podendo mais admitir uma prática docente que venha reforçar a passividade em vez de despertar a espontaneidade e o espírito de criatividade do aluno, visando à construção de um futuro melhor e o desenvolvimento sociocultural e econômico de cada país (BRIDI, 2004).

O desenvolvimento da iniciação científica permite que a Instituição; favoreça uma maior exposição dos melhores talentos dentre seus alunos. Outro diferencial privilegiado mostrado pela iniciação científica em relação ao estudante regular refere-se à chance de se entender precocemente de ciência atualizada, em face do convívio com pesquisadores experientes.

\section{PROGRAMAS DE INICIAÇÃO CIENTÍFICA DESENVOLVIDOS NAS INSTITUI- ÇÕES DE ENSINO SUPERIOR DO BRASIL}

Atualmente, há diversos programas de IC financiados pelo CNPq, destinados aos estudantes e professores do ensino superior, dentre eles podemos citar: PIBIC, PIBIC-AF, PIBITI, PICME, que serão descritos a seguir.

O Programa Institucional de Bolsas de Iniciação Científica (PIBIC) atende a instituições públicas e privadas e tem como foco principal promover uma ênfase 
científica aos novos talentos que estão para se formar, e ele é apoiado pelo CNPq com a concessão de bolsas. Os projetos de pesquisa nos quais os alunos participam devem ter qualidade acadêmica, mérito científico e orientação adequada por um professor - pesquisador (INPE, 2017).

O CNPq propõe os objetivos gerais do PIBIC, delineados nessa normatização, da seguinte forma:

1. Contribuir para a formação de recursos humanos para a pesquisa;

2. Contribuir de forma decisiva para reduzir o tempo médio de titulação de mestres e doutores;

3. Contribuir para que, na próxima década, diminuam as disparidades regionais na distribuição da competência científica do País;

4. Possibilitar maior interação entre graduação e pós-graduação;

5. Qualificar os melhores alunos para os programas de pós-graduação;

6. Incentivar talentos potenciais entre estudantes de graduação.

Em relação aos bolsistas, o PIBIC pretende: a) despertar vocação científica e incentivar talentos potenciais entre estudantes de graduação, mediante suas participações em projetos de pesquisa, introduzindo o jovem universitário no domínio do método científico; b) proporcionar ao bolsista, orientado por pesquisador qualificado, a aprendizagem de técnicas e métodos científicos, bem como estimular o desenvolvimento do pensar cientificamente e da criatividade, decorrentes das condições criadas pelo confronto direto como os problemas de pesquisa; c) possibilitar a diminuição do tempo de permanência do bolsista na pós-graduação; d) despertar no bolsista uma nova mentalidade em relação à pesquisa e prepará-lo para a pósgraduação (BRIDI, 2004).

O PIBIC nas Ações Afirmativas - PIBIC-AF é dirigido às universidades públicas que são beneficiárias de cotas PIBIC e que têm programa de ações afirmativas. Trata-se de um programa piloto que prevê a distribuição de IC às instituições que preencham esses requisitos e se interessem em participar do programa. O PIBIC-AF é um programa que tem como missão complementar as ações afirmativas já existentes nas universidades. Seu objetivo é oferecer aos alunos beneficiários dessas políticas a possibilidade de participação em atividades acadêmicas de iniciação científica 
(CNPq, 2017).

O Programa Institucional de Bolsas de Iniciação em Desenvolvimento Tecnológico e Inovação (PIBITI) tem por objetivo estimular os jovens do ensino superior nas atividades, metodologias, conhecimentos e práticas próprias ao desenvolvimento tecnológico e processos de inovação, bem como aperfeiçoar os recursos humanos que se dedicarão ao fortalecimento da capacidade inovadora das empresas no País, com condições de participar de forma criativa e empreendedora (CNPq, 2017).

O Programa de Iniciação Científica e Mestrado (PICME) oferece aos estudantes universitários que se destacaram nas Olimpíadas de Matemática (medalhistas da Olimpíada Brasileira de Matemática das Escolas Públicas (OBMEP) ou da Olimpíada Brasileira de Matemática (OBM)) a oportunidade de realizar estudos avançados em Matemática simultaneamente com sua graduação. O Programa visa fortalecer a área de matemática no País por meio da concessão de bolsa de Iniciação Científica aos estudantes que ingressaram na graduação, em qualquer área do conhecimento, e que foram medalhistas na OBMEP ou da OBM (CNPq, 2017).

Assim, as ações desenvolvidas pelo PIBIC centralizam-se na iniciação científica de novos talentos em todas as áreas do conhecimento, buscando a formação integral do aluno de graduação, garantindo a sua inserção nos espaços de ensino, pesquisa e extensão.

\section{A CONTRIBUIÇÃO DA INICIAÇÃO CIENTÍ́FICA PARA A FORMAÇÃO DO GRA- DUANDO}

Segundo Fernandes (2002), o aluno que desenvolve pesquisa na universidade tem a possibilidade de elaborar o seu próprio pensamento, seu conhecimento, ou seja, ao realizar pesquisas para descobrir, aprender ou criar novos conhecimentos, relaciona a teoria e a prática.

Para Favade-Moraes e Fava (2000) a IC é importante para o estudante pois melhora a sua análise crítica, dar-lhe maturidade intelectual, desenvolve sua compreensão em relação à ciência e concede possibilidades futuras, tanto acadêmicas 
quanto profissionais, em uma possível inserção na pós-graduação, bem como oferece auxílio financeiro, podendo ser utilizado na aquisição de livros e materiais acadêmicos ou como subsídio próprio.

Massi e Queiroz (2010) investigaram as contribuições de pesquisas sobre IC no Brasil, e os resultados apontaram para a existência de um amplo consenso sobre o papel relevante que essa atividade desempenha na formação dos graduandos, principalmente no que diz respeito às atividades realizadas no curso de graduação, ao desenvolvimento pessoal, à construção de uma nova visão de ciência e à socialização profissional.

Pachane (1998), no seu estudo sobre a contribuição da experiência universitária no desenvolvimento pessoal do aluno, verificou que, dentre os espaços de participação do estudante no contexto universitário, a realização de trabalhos de pesquisas é a que mais abre possibilidades ao aluno, sendo uma de suas principais satisfações, em especial pela possibilidade de realizar um trabalho prático, gratificante em termos pessoais e que pode ser remunerado.

As atividades relacionadas à pesquisa são consideradas fundamentais para a formação acadêmica, profissional e pessoal. Tais atividades proporcionam aos estudantes correlacionarem a teoria com a prática, e aprenderem a trabalhar com planejamento, organização e prazos, crescimento intelectual e adquirirem maturidade, assumindo a postura de cientistas. Nesta concepção a pesquisa é caracterizada como processo educativo emancipatório, para tanto a pesquisa precisa ser considerada como capacidade de questionamento, de elaboração e reelaboração constante da própria aprendizagem (FERNANDES, 2002).

\section{CONCLUSÕES}

Dessa forma conclui-se que a iniciação científica traz inúmeros benefícios aos jovens universitários, ao se constituir em um instrumento educativo possibilitando ao estudante a participação do processo de produção do conhecimento científico de sua área de formação.

Outro benefício de participar de um programa de IC é o de permitir a articulação entre os vários conhecimentos, constituindo um dos caminhos para a exe- 
cução de projetos interdisciplinares, que envolvam a relação entre Teoria e Prática.

A pesquisa científica proporciona ao estudante o desenvolvimento da capacidade de expressão e elaboração do conhecimento, despertando nele a curiosidade, criatividade e interesse pelo saber.

A IC é considerada uma experiência positiva para os estudantes, pois incentiva-os a encontrarem motivação para desenvolver resultados além do esperado, dando-lhes autonomia e proatividade no campo acadêmico. Assim, estes estudantes se destacam entre os demais e podem vir a apresentar melhores resultados em sua carreira profissional.

Geralmente os estudantes que participam da iniciação científica apresentam melhores desempenhos nas seleções para a pós-graduação, terminam mais rápido a titulação, possuem um treinamento mais coletivo, apresentam espírito de equipe e detêm mais facilidade de falar em público e de se adaptar às atividades didáticas futuras. Melhora-se o perfil do estudante, transformando-o de agente passivo para ativo. Lhe é permitida uma evolução que implicará em efeitos para a universidade e para a comunidade em que este estudante esteja inserido.

A iniciação científica também proporciona a possibilidade de o aluno publicar artigos em periódicos e revistas, apresentar pôsteres e trabalhos em semanas de divulgação científica, além de adquirir a importante experiência de ter seu desempenho avaliado por outros profissionais da área. Essa é uma oportunidade que muitos pesquisadores somente começam a ter após a graduação, quando decidem fazer um mestrado ou uma especialização. Quem começa nas atividades científicas desde a graduação se destaca da concorrência e se forma com um histórico de publicações muito mais vasto e complexo.

Vale destacar também que; a IC se revela um diferencial na trajetória profissional dos estudantes, pois representa um importante fator de contratação no ambiente de trabalho e de formação e encaminhamento para a pesquisa no ambiente universitário. A experiência acadêmica é carregada com ele perdurando e sendo repassada e reutilizada por outros que estão apenas iniciando essa fase.

\section{REFERÊNCIAS}

ÂNIMA Educação. Veja os principais benefícios da Iniciação Científica para o aluno. Disponível em: < http://blog.una.br/veja-os-principais-beneficios-da-iniciacao-cienti- 
fica-para-o-aluno/> Acesso em: 31 out. 2017.

BARIANI, I.C. Estilos Cognitivos de Universitários e Iniciação Científica. 1998. Tese (Doutorado em Educação) - Universidade Estadual de Campinas, 1998.

BAZIN, M.J. O Que é a iniciação científica. Revista de Ensino de Física, São Paulo, v. 5 , n. 1, p. $81-88$, jun. 1983 .

BRASIL. Lei no 9.394 de 20 de Dezembro de 1996. Dispõe sobre as Diretrizes e Bases da Educação Nacional. Disponível em: < http://www.planalto.gov.br/ccivil_03/leis/L9394.htm.> Acesso em: 31 out. 2017.

BRIDI, J.C. A. A Iniciação Científica na Formação do Universitário. 2004. $147 f$. Dissertação (Mestrado) - Universidade Estadual de Campinas, Campinas, 2004.

CAMPOS, F.G.G.; SANTOS, R.F.; SANTOS, F.C.P. A importância da pesquisa científica na formação profissional dos alunos do curso de educação física do Unileste-MG. MOVIMENTUM - Revista Digital de Educação Física, Ipatinga, v. 4, n. 2, ago./dez. 2009.

CNPq, Centro Nacional de Desenvolvimento Científico e Tecnológico. Iniciação Científica. Disponível em: < http://goo.gl/W8pOJz> . Acesso em: 23 out. 2017.

INPE - Instituto Nacional de Pesquisas Espaciais. O que é PIBIC? Disponível em: < http://www.inpe.br/bolsas/pibic/index.php.> Acesso em: 31 out. 2017.

DELIBERAÇÃO CONSU-A-24, de 30/09/03 Do Fundo de Apoio ao Ensino, Pesquisa e Extensão - FAEPEX. Disponível em: < https://www.prp.unicamp.br/pt-br/deliberacao-consu-0242003>. Acesso em: 29 out. 2017.

FAVA-DE-MORAES, F.; FAVA, M. A iniciação científica: muitas vantagens e poucos riscos. São Paulo em Perspectiva, v. 14, n. 1, p. 73-77, jan./mar. 2000.

FERNANDES, M. C. S. G. Concepções de qualidade de ensino na perspectiva docente em um centro universitário privado e noturno. Tese (Doutorado em 
Educação) - UNESP, Araraquara, 2002.

FIOCRUZ, Fundação Oswaldo Cruz. O que é Iniciação científica. Disponível em: $<$ http://www.juventudect.fiocruz.br/iniciacao-cientifica/o-que-e-iniciacao-cientifica > . Acesso em: 30 out. 2017.

GRAY, D. E. Doing research in the real world. 3. ed. Londres: Sage, 2014.

LOPES, N. D. Importância da Iniciação Científica na Universidade. Reverso Online. Bahia, mar. 2014. Disponível em: < https://www3.ufrb.edu.br/reverso/importanciada-iniciacao-cientifica-na-universidade/> . Acesso em: 28 mar. 2018.

MASSI, L.; QUEIROZ, S. L. Estudos sobre Iniciação Científica no Brasil: uma revisão. Cadernos de Pesquisa, v. 40, n. 139, p. 173-197, jan./abr 2010.

MASSI, L.; QUEIROZ, S. L. Pesquisas sobre Iniciação Científica no Brasil: características do seu desenvolvimento nas universidades e contribuições para os graduandos. Revista Brasileira de Iniciação Científica, v. 1, n. 1, p. 38-64, 2014.

MASSI, L.; QUEIROZ, S. L. Iniciação científica: aspectos históricos, organizacionais e formativos da atividade no ensino superior brasileiro. São Paulo: Ed. da Unesp digital, 2015.

OLIVEIRA, C.S. A Importância da Iniciação Científica para a Universidade, para o Estudante e para a Comunidade. 2008. Disponível em: < https://cassioso.wordpress.com/importancia-da-iniciacao-cientifica/> . Acesso em: 31 out. 2017.

OLIVEIRA, L.C.V. Iniciação à pesquisa no ensino superior: o novo e o velho espírito científico nas atividades acadêmicas. Reunião Anual da ANPED, 2001.

PACHANE, G.G. A Universidade vivida: a experiência universitária e sua contribuição ao desenvolvimento pessoal a partir da perspectiva do aluno. 1998. Dissertação (Mestrado em Educação) - Universidade Estadual de Campinas, Campinas, 1998.

PELUSO, D.L. O Incentivo a pesquisa científica na formação inicial de professores 
de educação física. In: XVIII CONGRESSO BRASILEIRO DE CIÊNCIAS DO ESPORTE (CONBRACE), 18., V CONGRESSO INTERNACIONAL DE CIÊNCIAS DO ESPORTE (CONICE), 5. Anais... [s.l.; s.n.], 2013.

PIMENTA, S.G. Docência no ensino superior. São Paulo: Cortez, 2005.

PINTO, N.L.S.; FERNANDES, L.M.A.; SILVA, F.F. Para além da formação acadêmica: As contribuições da iniciação científica para o desenvolvimento pessoal e profissional de estudantes da área de administração. Administração: Ensino \& pesquisa, Rio de Janeiro v. 17, n. 2, p. 301-325, 2016.

SANCHES, C. O desafio de fazer pesquisa científica no Brasil. LabNetwork, São Paulo Ago. 2016. Disponível em: < http://www.labnetwork.com.br/especiais/o-desafiode-fazer-pesquisa-cientifica-no-brasil/> . Acesso em: 28 mar. 2018.

SANTOS, E. A. Produção científica: uma análise de sua contribuição na formação acadêmica e profissional dos discentes de ciências contábeis da UEFS. 2011. Trabalho de Conclusão de Curso (monografia) - Universidade Estadual de Feira de Santana, 2011.

SANTOS, E. S. O Professor como Mediador no Processo Ensino Aprendizagem. Revista Gestão Universitária, n. 40. Disponível em: < http://www.udemo.org.br/RevistaPP_02_05Professor.>. Acesso em: 30 out. 2017.

SANTOS, A.C.; FERREIRA, F.L. Importância da pesquisa como princípio educativo para a formação científica de educandos do ensino superior. Caderno Intersaberes, v. 3, n. 4, p. 70-80, 2014.

SILVA, L. F. F. Iniciação científica: contexto e aspectos práticos. Rev Med., São Paulo. v. 91, n. 2, p. 128-36, abr./jun. 2012.

SIMÃO, L. M. et al. O Papel da iniciação científica para a formação em pesquisa na pós-graduação. In: SIMPÓSIO DE PESQUISA E INTERCÂMBIO CIENTÍFICO DA ASSOCIAÇÃO NACIONAL DE PESQUISA E PÓS-GRADUAÇÃO EM PSICOLOGIA, 6., 1996. Rio de Janeiro. Anais... Rio de Janeiro: ANPPEP, 1996. p. 111-113. 
SHIGUNOV NETO, A.S.; MACIEL, L. S. B. A Importância da pesquisa para a prática pedagógica dos professores que atuam na educação superior brasileira: algumas discussões iniciais. Revista Brasileira de Docência, Ensino e Pesquisa em Administração, v. 1, n. 1, p. 4-23, 2009.

Recebido em: 21/11/2017

Aceito em: 16/04/2018 